\title{
A novel torque sensor based on the angle of magnetization vector
}

\author{
Yinguo Huang, Yong Yang, Xiaomei Zhang and Meirong Zhao*
}

\begin{abstract}
Torque is an important parameter of a mechanical power system, which reflects transmission efficiency, transmission reliability, and operating conditions of equipment. Torque monitoring is very important for real-time control and fault diagnosis of mechanical equipment. A torque measurement method based on the theory of magnetic effect is proposed for multipoint torque monitoring in shafting. A model of the deflection angle of the magnetization vector and torque is established based on the theory of magnetic equivalent. A non-contact signal extraction circuit based on hall sensor is designed, a torque loading experimental device is set up, and the torque calibration experiment is completed. The experimental results show that the sensitivity of the torque measurement system is $17.7 \mathrm{mV} / \mathrm{Nm}$, and the maximum nonlinear error is $0.77 \%$ full scale. The deflection angle of the magnetization vector has a good linear relation with the torque, which can be measured indirectly by the deflection angle. This method has the advantages of a simple device, strong anti-interference ability, and non-contact measurement. Because it is not necessary to do special treatment to the elastic shaft, it is convenient to form a non-contact torque sensing node, which can realize real-time monitoring of multipoint torque of shafting.
\end{abstract}

Keywords: Torque measurement, The deflection angle, Magnetization vector, Multipoint torque monitoring, Non-contact measurement

\section{Introduction}

The drive shaft is very important in many fields, such as robotic design, force measurement system, and vessel [1-5]. It is an indispensable part of an automotive transmission system with the feature of high rotation speed, and less bracing transmits torque in the operation of a vehicle. When the vehicle starts, runs, decelerates, and brakes, the greater the torque of the shaft is, the more serious it has an influence on the performance of the vehicle. Accordingly, real-time monitoring of the status of the torque of the shaft has a great influence on the finding out the faults of the drive shaft in time.

Signal communication is a big problem in the measurement of rotational torque. There are two main ways to transmit the measured signal when the elastic shaft is running at high speed. Firstly, the wireless communication unit is pasted on the surface of the elastic shaft. In

\footnotetext{
* Correspondence: meirongzhao@tju.edu.cn

State Key Laboratory of Precision Measuring Technology and Instruments, Tianjin University, Tianjin 300072, China
}

this way, the installation of a wireless communication unit is strict, and the volume should not be too large. Because of the load effect, the attached wireless communication equipment will affect the moment of inertia of the shaft, and the mass of the axle is eccentric in the case of high-speed rotation, which will affect the measurement precision of the shaft torque. Secondly, a novel photoelectric, magnetoelectric, and capacitive torque sensor is used to measure the rotational torque. For example, a novel photoelectric torque sensor for highspeed rotating machinery on the basis of adopting photoelectric reflex measuring method is proposed; the sensor is based on phase measurement principle; the laser head and the reflective stripes are as transmission devices; it can measure the rotating machines torque under the condition of high speed [6]. A new kind of magnetoelectric non-contact torque sensor is composed of a special ring space and a special magnetoelectric detector array. According to the principle of magnetoelectric induction, the sine signals with a certain frequency and amplitude are imposing on the excitation coils, 
when there is torque on the mechanical shaft, the magnetoelectric detector will respond displacement between the annular narrow and the ring array, the secondary coil can generate induction signal which relative to the excitation [6].

The magnetic torque sensor has many advantages, such as durability, wide application, high reliability, and good prospects for development. It is widely concerned by researchers. ShaoPeng studied a ring torque sensor [7] based on the counter magnetostriction effect of amorphous alloy. It used the method of sticking the ribbon of the amorphous alloy. However, it was large, not easy to install, and not easy to monitor the torque in real time. Meng et al. studied a non-contact torque sensor [8] based on the change of magnetic induction, which was not suitable for dynamic measurement of torque because it needed manually adjusting two digital potentiometers. Muro et al. studied a ring torque sensor [9] based on the measurement of magnetic field by the Hall detection circuit, and magnetostrictive effect whose measuring range was small. Jiles studied the theory of magnetomechanical effects [10] and it provided a theoretical basis for the magnetic torque sensor. Sablik et al. studied the magnetostriction when the magnetic shaft suffered a tensile or compressive stress and applied a magnetic field. The stress and magnetic field were coaxial [11]. The torque sensor proposed in this paper uses the non-contact signal extraction circuit to detect the deflection angle of magnetization vector and explores the relationship between the deflection angle and the torque; instead of using the wireless communication equipment, the non-contact torque measurement is realized directly.

\section{The model of torque measurement}

The distribution of magnetization at a point at the surface of a torsional circular shaft is shown in Fig. 1. The

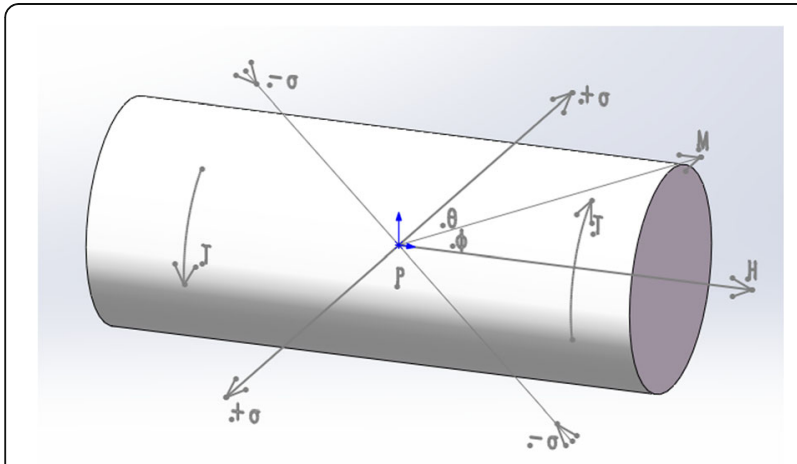

Fig. 1 The distribution of magnetization at a point on the outer surface of the shaft magnetic induction intensity along the axis direction can be expressed by the following formula [12-14].

$$
B=\mu_{0}(H+M \cos \phi)
$$

$B$ is the magnetic induction intensity along the axis direction, $\mu_{0}$ is the vacuum permeability, $H$ is the external magnetic field, $M$ is the magnetization, and $\phi$ is the deflection angle of the magnetization vector.

The effect of torque on the circular shaft can be equivalent to the tension or compression stress acted on the shaft with a $45^{\circ}$ angle to the axis. The equivalent tension and compression stress are proportional to the applied torque, which can be expressed with the following formula:

$$
\sigma=\frac{16 T}{\pi D^{3}}
$$

where $\sigma$ is the equivalent stress, $T$ is the applied torque, and $D$ is the diameter of the circular shaft.

According to the theory of magnetomechanical effects [4], the stress on a circular shaft can be equivalent to the effective magnetic field $H_{\sigma}$ on a circular shaft, so $M$ is equal to the combined vector of $H$ and $H_{\sigma}$. When it comes to a shaft with positive magnetostrictive effect, the direction of the magnetization vector is near the direction of the tensile stress, $+\sigma$, and away from the direction of the compressive stress, $-\sigma$, as shown in Fig. 1. The offset of magnetization is invariable under the action of constant external magnetic field. There is a thermodynamic equilibrium relationship which can be expressed with the following formula:

$$
\begin{aligned}
\Omega= & U-\mu_{0} H M \cos \left(\frac{\pi}{4}-\theta\right)-\frac{3}{2} \sigma \lambda(\sigma) \\
& \times\left(\cos ^{2} \theta-v \sin ^{2} \theta\right)-\frac{3}{2}(-\sigma) \lambda(-\sigma) \\
& \times\left(\sin ^{2} \theta-v \cos ^{2} \theta\right)
\end{aligned}
$$

where $U$ is the energy density produced by the intercoupling of magnetic domains within the circular shaft, the second item is magnetostatic energy density, the last two items are the magnetic elastic coupling energy density produced by circular shaft torsion, $\theta$ is the angle between the equivalent tensile stress and the magnetization, $v$ is Poisson ratio, and $\lambda(\sigma)$ represents the magnetostriction produced by the uniaxial tensile stress and the external magnetic field when they are coaxial. $\lambda(-\sigma)$ represents the magnetostriction produced by the uniaxial compressive stress and the external magnetic field when they are coaxial. $\lambda$ can be expressed with the following formula: 


$$
\lambda=\gamma_{1} M^{2}+\gamma_{2} M^{4}
$$

$\gamma_{1}$ and $\gamma_{2}$ are related to the stress, they can be expressed with the following formula:

$$
\begin{aligned}
& \gamma_{1}=\gamma_{11}+\gamma_{12} \sigma \\
& \gamma_{2}=\gamma_{21}+\gamma_{22} \sigma
\end{aligned}
$$

$\gamma_{11}, \gamma_{12}, \gamma_{21}$, and $\gamma_{22}$ are constants related to the material of the shaft.

When it comes to the shaft whose material is positive magnetostrictive, $\Omega$ increases with the increase of $\theta$. That means the equivalent tensile stress will make the direction of magnetization close to the direction of tensile stress, and the equivalent compressive stress will make the direction of magnetization vector approaching to the direction of compressive stress under the constant external magnetic field. Accordingly, the equilibrium condition is $d \Omega / d \theta=0$, and then, the following formula can be obtained.

$\frac{1}{\sqrt{2}} \mu_{0} H M(\sin \theta-\cos \theta)+\frac{3}{2} \sigma(1+v) \sin 2 \theta(\lambda(\sigma)+\lambda(-\sigma))=0$

According to $\theta+\phi=\pi / 4$ and $\cos 2 \phi=\cos ^{2} \phi-\sin ^{2} \phi$, Formula (7) can be converted to a quadratic equation with one unknown whose independent variable is $\sin \phi$.

$$
2 C \sin ^{2} \phi+\sin \phi-C=0
$$

The expression of the deflection angle of the magnetization vector $\phi$ can be obtained by solving Formula (8),

$$
\phi=\sin ^{-1} \frac{-1+\sqrt{1+8 C^{2}}}{4 C}
$$

where $C=\frac{3}{2} \frac{\sigma(1+v)}{\mu_{0} H M}(\lambda(\sigma)+\lambda(-\sigma))$. A model between the deflection angle of the magnetization vector and the torque $T$ has been established, combined with the above equation.

\section{Methods}

\subsection{Experimental facility}

The torque measurement experimental device includes a torque loading device, Hall detection circuit, shaft, oscilloscope, DC power supply, and so on. The setup is shown in Fig. 2. The material of the shaft is carbon steel, and the diameter of the shaft is $20 \mathrm{~mm}$. The permanent magnet ring magnetized the shaft.

\subsection{Torque loading device}

The torque loading device is designed to simulate the process of the action of the torque on the drive shaft. The shaft end is fixed on the precision platform, and

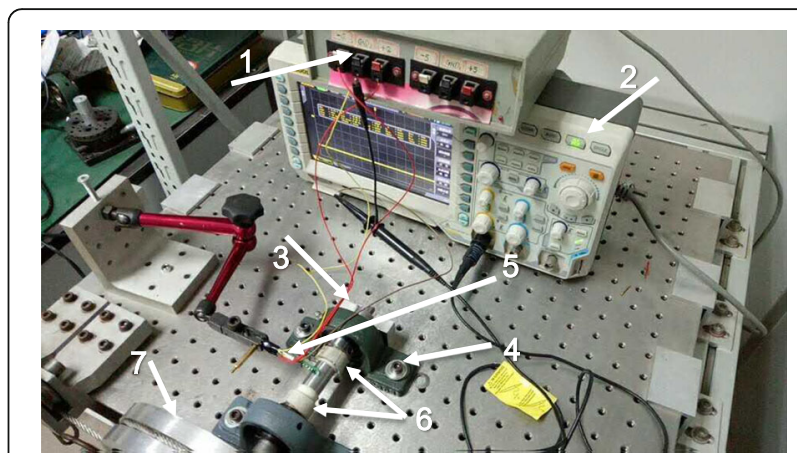

Fig. 2 The torque measurement device. (1) DC power supply, (2) oscilloscope, (3) fixed parts, (4) bearing, (5) non-contact signal extraction circuit, (6) permanent magnet ring, and (7) torque loading device

the other end is connected with the flange plate used to exert torque through the bearing. The torque of the drive shaft is simulated by applying the same weight at both ends of the flange plate. This torque loading device can effectively reduce the effect of bending moment.

\subsection{Non-contact signal extraction circuit}

The Hall detection circuit is designed to detect the deflection angle of the magnetization vector of the magnetic iron shaft under the action of torque. The Hall circuit needs a voltage stabilizer to supply the power. In addition, the output voltage is so weak that the detection and processing of the signal are very difficult. Hence, an amplifier with high common mode rejection ratio is adopted. The amplifier can change the different magnification by adjusting the size of the adjustable resistance.

The Hall detection circuit was calibrated by the high-precision angle stage. The formula between the deflection angle, $\theta$, and the output voltage, $V$, can be obtained by the least square method to fit the straight line. They can be expressed by Formula (10). The maximum error value of the detection circuit is $6.97 \mathrm{~min}$, and the sensitivity is $25.476 \mathrm{mV} / \mathrm{min}$.

$$
V=25.476 \theta-10662(\mathrm{mV})
$$

\subsection{Experimental process}

Firstly, complete the construction of the experimental equipment, which is shown in Fig. 2. Secondly, applying the same weight at both ends of the flange plate is equivalent to the torque applied to the shaft. In the experiment, the applied torque is $9.71 \mathrm{Nm}, 19.02 \mathrm{Nm}$, $28.32 \mathrm{Nm}, 37.59 \mathrm{Nm}, 46.88 \mathrm{Nm}, 56.13 \mathrm{Nm}, 65.48 \mathrm{Nm}$, $74.76 \mathrm{Nm}, 84.05 \mathrm{Nm}$, and $93.35 \mathrm{Nm}$. The torque can change the deflection angle of the magnetization vector 


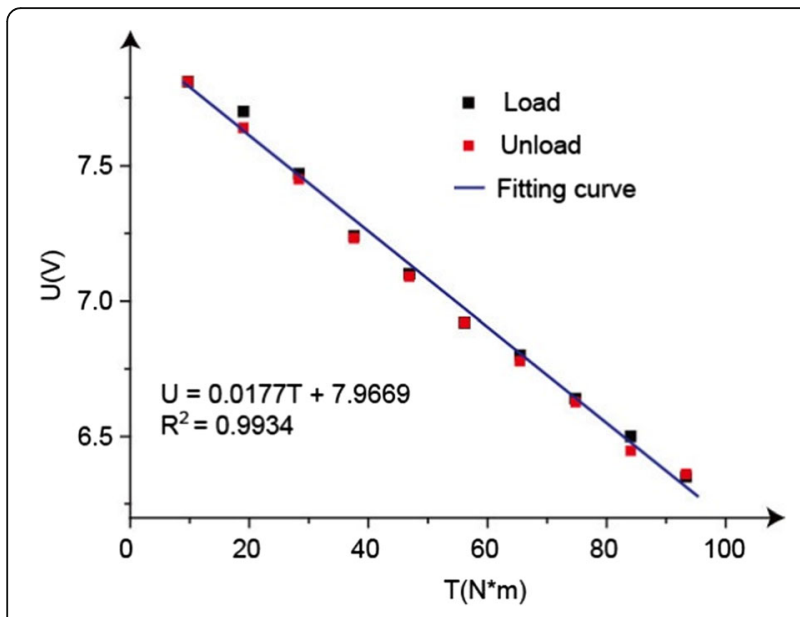

Fig. 3 The output of the torque sensor

of the shaft, and then, the non-contact signal extraction circuit can detect the deflection angle. The deflection angle of the magnetization vector under the corresponding torque action can be obtained from Formula (10). Finally, the relation between torque and the deflection angle of the magnetization vector can be obtained.

\section{Results}

\subsection{Loading and unloading experiments}

In loading and unloading experiments, torque is applied to the shaft by increasing weights, and then, the weight is reduced in turn, and each output voltage of Hall circuit is recorded. The fitting curves are drawn according to the experimental data, and the relationship between the torque and the output voltage is obtained, as shown in Fig. 3. The output voltage and torque are approximately linear both in the loading process and in the unloading process. They can be expressed by Formula (11).

$$
V=0.0177 T+7.9669
$$

It means that the output voltage is proportional to the torque in the full scale. The maximum nonlinear error is $0.77 \%$ full scale, and the sensitivity is $17.7 \mathrm{mV} / \mathrm{Nm}$.

\subsection{Repeatability experiment}

In the repeated experiment, the torque is constantly increased and the output voltage is recorded. Repeat the experiment six times. The repeatability experiment data are shown in Table 1 . The repeatability can be expressed by Formula (12).

$$
s=\sqrt{\frac{\sum_{i=1}^{n}\left(x_{i}-\bar{x}\right)^{2}}{n-1}}
$$

$s$ is the measurement repeatability, $x_{i}$ is the measured data every time, $\bar{x}$ is the average, and $n$ is the experiment times.

The data in the table shows that the maximum repeatability is $4.65 \%$. The main influence factors are the phenomenon of magnetic hysteresis of the magnetized shaft in the combined action of the torque and external magnetic field.

\section{Discussion}

The method proposes a new idea to measure the torque. The results show that the torque is linear to the deflection angle of the magnetization vector. There are some ways to improve this method, which could get better results and make the measurement results more accurate [15-20].

(1) Torque loading device

\begin{tabular}{|c|c|c|c|c|c|c|c|}
\hline Torque (Nm) & $\begin{array}{l}\text { First measured } \\
\text { data }(V)\end{array}$ & $\begin{array}{l}\text { Second measured } \\
\text { data }(V)\end{array}$ & $\begin{array}{l}\text { Third measured } \\
\text { data }(V)\end{array}$ & $\begin{array}{l}\text { Fourth measured } \\
\text { data }(V)\end{array}$ & $\begin{array}{l}\text { Fifth measured } \\
\text { data }(V)\end{array}$ & $\begin{array}{l}\text { Sixth measured } \\
\text { data }(V)\end{array}$ & Repeatability (\%) \\
\hline 9.71 & 7.80 & 7.81 & 7.86 & 7.80 & 7.80 & 7.80 & 2.40 \\
\hline 19.02 & 7.65 & 7.70 & 7.67 & 7.67 & 7.65 & 7.65 & 1.97 \\
\hline 28.32 & 7.43 & 7.47 & 7.48 & 7.53 & 7.45 & 7.48 & 3.39 \\
\hline 37.59 & 7.24 & 7.24 & 7.28 & 7.36 & 7.25 & 7.25 & 4.65 \\
\hline 46.88 & 7.11 & 7.10 & 7.10 & 7.16 & 7.10 & 7.10 & 2.40 \\
\hline 56.13 & 6.96 & 6.92 & 6.95 & 7.00 & 6.95 & 6.96 & 2.58 \\
\hline 65.48 & 6.82 & 6.80 & 6.87 & 6.82 & 6.82 & 6.84 & 2.40 \\
\hline 74.76 & 6.68 & 6.64 & 6.72 & 6.72 & 6.68 & 6.68 & 3.01 \\
\hline 84.05 & 6.52 & 6.50 & 6.53 & 6.60 & 6.54 & 6.54 & 3.37 \\
\hline 93.35 & 6.32 & 6.35 & 6.38 & 6.40 & 6.35 & 6.38 & 2.88 \\
\hline
\end{tabular}

Table 1 Repeatability experiment data 
The accuracy of torque provided by a torque loading device has a great impact on the experimental results. Therefore, a more precise torque loading device is needed. At the same time, the error compensation should be taken into consideration.

\section{(2) Non-contact signal extraction circuit}

The non-contact signal extraction circuit is used to detect the deflection angle of the magnetization vector. The measurement accuracy of the deflection angle has a great influence on the experimental results. The feedback circuit can be designed to enhance the stability of the circuit and reduce the measurement error.

\section{(3) Hysteresis effect}

Hysteresis effect has great influence on the linearity of the system. Hysteresis on system error should be taken into consideration, and the model of torque measurement should be revised.

\section{Conclusions}

The magnetic torque sensor mostly measures the amplitude and explores the relationship between the amplitude and the torque. The torque measurement method proposed in this paper is detecting the deflection angle of the magnetization vector. The measurement of amplitude is easily affected by various factors, but the deflection angle of the magnetization vector has a strong anti-interference ability and is not easy to change. It can enhance the reliability of torque measurement and ensure the accuracy of measurement.

The model of torque measurement is derived based on the thermodynamic equilibrium. A torque loading device and a Hall detection circuit are designed to test the model. The experimental results show that the sensitivity of the torque measurement system is $17.7 \mathrm{mV} / \mathrm{Nm}$, and the maximum nonlinear error is $0.77 \%$ full scale. The experimental results verify the feasibility of this torque measurement method.

\section{Acknowledgements}

The authors would like to thank the reviewers for their thorough reviews and helpful suggestions.

\section{Funding}

This work is supported by the National Natural Science Foundation of China (Grant No 61304246).

\section{Authors' contributions}

YH is the main writer of this paper. He proposed the main idea. YY completed the experiment, analyzed the result, and finished the manuscript. $\mathrm{XZ}$ designed the non-contact signal extraction circuit. MZ gave some important suggestions. All authors read and approved the final manuscript.

\section{Publisher's Note}

Springer Nature remains neutral with regard to jurisdictional claims in published maps and institutional affiliations.

Received: 10 February 2018 Accepted: 18 September 2018

Published online: 26 September 2018

\section{References}

1. Y.L. Zheng, H.Y. Lu, W. Yin, D.S. Tao, L.C. Shi, Y. Tian, Elegant shadow making tiny force visible for water-walking arthropods and updated Archimedes' principle. Langmuir 32, 10522-10528 (2016)

2. Y. Zheng, L. Song, G. Hu, et al., The multi-position calibration of the stiffness for atomic-force microscope cantilevers based on vibration. Meas. Sci. Technol. 26(5), 055001 (2015)

3. G. Zhang, G. Xie, L. Si, S. Wen, D. Guo, Ultra-low friction self-lubricating nanocomposites with mesoporous metal-organic frameworks as smart nanocontainer for lubricants. ACS Appl. Mater. Interfaces 9, 38146-38152 (2017)

4. J. Li, J. Luo, Nonlinear frictional energy dissipation between silica-adsorbed surfactant micelles. J. Phys. Chem. Lett. 8, 2258-2262 (2017)

5. C. Dong, C. Yuan, X. Bai, J. Li, H. Qin, X. Yan, Coupling mechanism between wear and corrosion processes of 304 stainless steel in hydrogen peroxide environments. Sci. Rep. 7, 2327 (2017)

6. H. Zhao, Present situation and development review of torque measurement Appl. Mech. Mater. 422(422), 141-145 (2013)

7. S.P. Chen, Research on ring type torque sensor based on magnetoelastic effect of amorphous alloy (China, China University of Mining and Technology, 2016) (in Chinese)

8. R. Meng, H.Q. Wang, Signal acquisition and processing of non-contact torque sensor for vehicle. Control Eng. China. 19(2),157-160 (2012)

9. H. Muro, C. Saito, M. Shimada, et al., in Sensors. Magnetostrictive-ring type torque sensor using two Hall ICs with differential magnetic field detection (IEEE, 2014), pp. 412-415

10. D.C. Jiles, Theory of the magnetomechanical effect. J. Phys. D Appl. Phys. 28(8), 1537 (1995)

11. M.J. Sablik, D.C. Jiles, Modeling effects of varying torsion in magnetized steel. IEEE Trans. Magn. 36(5), 3248-3250 (2002)

12. O.M. Vasilevskyi, Metrological characteristics of the torque measurement of electric motors. Int. J. Metrol. Qual. Eng. 8(7), 9 (2017)

13. Y.L. Guo, Z.F. Chang, N.A. Shao-Dan, Magnetic field and output voltage analysis of 3D torque sensor. Transducer Microsyst. Technol. 36(2),18-20 (2017)

14. J.C.S. Borges, D.B.B.D. Deus, A.C.L. Filho, et al., New contactless torque sensor based on the Hall effect. IEEE Sensors J. PP(99), 1 (2017)

15. K.C. Min, J.Y. Choi, S.Y. Sung, et al., Torque analysis of magnetic spur gear with radial magnetized permanent magnets based on analytical method. Trans. Korean Inst. Electr. Eng. 64(4), 545-551 (2015)

16. Q. Liang, X. Cheng, S.C.H. Huang, et al., Radar and sonar sensor networks. EURASIP J. Wirel. Commun. Netw. 2010(1), 948604 (2010)

17. Q. Liang, Radar sensor networks: algorithms for waveform design and diversity with application to ATR with delay-doppler uncertainty. EURASIP J. Wirel. Commun. Netw. 2007(1), 1-9 (2007)

18. Q. Liang, Biologically inspired target recognition in radar sensor networks. EURASIP J. Wirel. Commun. Netw. 2009(1), 1-8 (2010)

19. X. Li, Q. Liang, F.C. Lau, A maximum likelihood routing algorithm for smart grid wireless network. EURASIP J. Wirel. Commun. Netw. 2014(1), 75 (2014)

20. J. Chen, Q. Liang, Rate distortion performance analysis of nested sampling and coprime sampling. EURASIP J. Adv. Signal Process. 2014(1), 1-8 (2014) 